\title{
The Development and Characterization of an scFv-Fc Fusion-Based Gene Therapy to Reduce the Psychostimulant Effects of Methamphetamine Abuse
}

\author{
Charles E. Hay, Laura E. Ewing, Michael D. Hambuchen, Shannon M. Zintner, \\ Juliana C. Small, Chris T. Bolden, William E. Fantegrossi, Paris Margaritis, \\ S. Michael Owens, and Eric C. Peterson \\ University of Arkansas for Medical Sciences, Little Rock, Arkansas (C.E.H., L.E.E., M.D.H., C.T.B., W.E.F., S.M.O., E.C.P,); The \\ Children's Hospital of Philadelphia, Philadelphia, Pennsylvania (S.M.Z., J.C.S., P.M.)); The Raymond G. Perelman Center for \\ Cellular and Molecular Therapeutics, The Children's Hospital of Philadelphia, Philadelphia, Pennsylvania (P.M.); and Department \\ of Pediatrics, The University of Pennsylvania, Perelman School of Medicine, Philadelphia, Pennsylvania (P.M.)
}

Received July 9, 2019; accepted March 31, 2020

\begin{abstract}
Methamphetamine (METH) continues to be among the most addictive and abused drugs in the United States. Unfortunately, there are currently no Food and Drug Administration-approved pharmacological treatments for METH-use disorder. We have previously explored the use of adeno-associated viral (AAV)mediated gene transfer of an anti-METH monoclonal antibody. Here, we advance our approach by generating a novel antiMETH single-chain variable fragment (scFv)-Fc fusion construct (termed 7F9-Fc) packaged into AAV serotype 8 vector (called $\mathrm{AAV}-\mathrm{scFv}-\mathrm{Fc}$ ) and tested in vivo and ex vivo. A range of doses $\left[1 \times 10^{10}, 1 \times 10^{11}\right.$, and $1 \times 10^{12}$ vector copies (vcs)/mouse] were administered to mice, eliciting a dose-dependent expression of 7F9Fc in serum with peak circulating concentrations of 48,1785 , and $3831 \mu \mathrm{g} / \mathrm{ml}$, respectively. Expressed 7F9-Fc exhibited high-affinity METH binding, $\mathrm{IC}_{50}=17 \mathrm{nM}$. Between days 21 and 35 after vector administration, at both $1 \times 10^{11} \mathrm{vc} /$ mouse and $1 \times 10^{12} \mathrm{vc} /$ mouse doses, the AAV-7F9-Fc gene therapy significantly decreased the
\end{abstract}

potency of METH in locomotor assays. On day 116 post-AAV administration, mice expressing 7F9-Fc sequestered over 2.5 times more METH in the serum than vehicle-treated mice, and METH concentrations in the brain were reduced by 1.2 times the value for vehicle mice. These data suggest that an AAVdelivered anti-METH Fc fusion antibody could be used to persistently reduce concentrations of METH in the central nervous system.

\section{SIGNIFICANCE STATEMENT}

In this manuscript, we describe the testing of a novel antimethamphetamine (METH) single-chain variable fragment-Fc fusion protein delivered in mice using gene therapy. The results suggest that the gene therapy delivery system can lead to the production of significant antibody concentrations that mitigate METH's psychostimulant effects in mice over an extended time period.

\section{Introduction}

Methamphetamine (METH)-use disorder (MUD) has been increasing yearly since at least 2008 , and its use as an illicit drug is second only to opioid abuse (Winkelman et al., 2018; Artigiani et al., 2019). Recovery from MUD is difficult, with rates of relapse being over $60 \%$ within the 1 st year of abstinence (Brecht and Herbeck, 2014). One pharmacological approach to aid in the recovery from MUD is to use antiMETH monoclonal antibodies (mAbs) to block or blunt METH

The project described was supported by the National Insitutes of Health (NIH): National Institute on Drug Abuse (NIDA) [R01-DA036600] and the National Institute for General Medical Sciences (NIGMS) [T32-GM106999 and R25-GM083297].

Financial disclosures: S.M.O. has financial and fiduciary interests in InterveXion Therapeutics LLC, a biopharmaceutical company. The University of Arkansas for Medical Sciences has licensed intellectual property developed by S.M.O. to InterveXion Therapeutics Therapeutics LLC.

https://doi.org/10.1124/jpet.119.261180. effects. Rather than targeting the sites of METH's action in the brain, these antibodies could act as pharmacokinetic antagonists, sequestering METH in the blood and reducing the amount reaching the brain (McMillan et al., 2002). MAb therapy could be used in conjunction with behavioral therapies. For example, if METH use occurs during the course of therapy, anti-METH mAbs, by either preventing or greatly decreasing the direct effects of METH, could improve the chances of patients continuing with MUD therapy rather than relapsing.

Delivery of the mAb by gene therapy could greatly extend the duration of action, reduce dosing frequencies, and reduce the impact of potential noncompliance issues. Adeno-associated viruses (AAVs) are commonly used as gene therapy vectors because they are nonpathogenic viruses that can deliver up to $4.5 \mathrm{~kb}$ of DNA to host tissues (Zolotukhin, 2005). Previous studies in mice have shown that a single dose of AAV delivering DNA encoding an entire mAb sequence reaches maximal expression of $\mathrm{mAb}$ in 3 to 4 weeks, and expression persists for

ABBREVIATIONS: AAV, adeno-associated viral; HRP, horseradish peroxidase; mAb, monoclonal antibody; METH, methamphetamine; MUD, METH-use disorder; scFv, single-chain variable fragment; vc, vector copy. 
at least 8 months (Hicks et al., 2012; Rosenberg et al., 2012, 2013; Hay et al., 2018). Hicks et al. (2012) showed that an antinicotine AAV-based mAb therapy elicited over 4-fold greater circulating concentrations of high-affinity mAbs with one injection compared with an antinicotine vaccine in rodents, which generated variable amounts of polyclonal antibodies and required two additional booster doses. These previous studies suggest that, in comparison with a METH vaccine, an optimized anti-METH gene therapy should be able to sequester greater levels of METH in the serum after only one injection without the need for boosters.

Other laboratories have successfully developed AAV-based $\mathrm{mAb}$ therapies against various drugs of abuse (Hicks et al., 2012; Rosenberg et al., 2012). A full IgG antibody sequence can fit into an AAV capsid but requires post-translational autocleavage of the heavy and light chains of the IgG antibody before dimerizing back together through disulfide bonds (Fang et al., 2005; Ho et al., 2013). In contrast, we have previously reported the design and testing of a single-chain variable fragment ( $\mathrm{scFv}$ ) 7F9, which consists of the variable regions of an IgG connected by a 15-amino acid linker (Hay et al., 2018). ScFv7F9 binds with high affinity to $\operatorname{METH}\left(\mathrm{K}_{\mathrm{D}}=6.2 \mathrm{nM}\right)$ and to the active metabolite amphetamine with lower affinity $\left(\mathrm{IC}_{50}=8 \mu \mathrm{M}\right)$ (Nanaware-Kharade et al., 2015). The short half-life of scFvs, which is approximately 60 minutes because of high clearance rate, limits the circulating concentrations of anti-METH antibodies in mice (NanawareKharade et al., 2015; Reichard et al., 2016; Hay et al., 2018).

An improvement in $\mathrm{scFv}$ therapeutic antibody properties was implemented using an $\mathrm{scFv}-\mathrm{Fc}$ fusion protein. The scFvFc fusion possesses much of the simplicity of an $\mathrm{scFv}$ consisting of one amino acid sequence but has an increased half-life of about 108 hours (Unverdorben et al., 2016). An scFv-Fc fusion consists of an $\mathrm{scFv}$ connected to the $\mathrm{C}_{\mathrm{H} 2}$ and $\mathrm{C}_{\mathrm{H} 3}$ regions of an IgG constant region (Repp et al., 2011), which can then create a homodimer with another copy of itself. An scFv-Fc fusion has the potential to avoid the more complex post-translational processing that is necessary for IgG assembly with a much longer half-life than an scFv. ScFv-Fc fusions are reported to have serum half-lives of 104-112 hours compared with the 218-222 hours of a full IgG antibody (Unverdorben et al., 2016). This led to our hypothesis that the inclusion of the Fc region from an IgG antibody forming an scFv-Fc fusion antibody against METH would yield high circulating concentrations. These high concentrations of the novel antibody should sequester greater concentrations of METH in the circulation and significantly reduce the psychostimulant effects of METH in mice over significant time periods.

In this study, we report a newly designed anti-METH scFvFc biotherapeutic (7F9-Fc) packaged into an AAV8 viral vector. We report a dose-dependent relationship between the AAV8 viral vector copies administered and the concentration of 7F9-Fc in the serum of mice. A $1 \times 10^{12}$ vector copy (vc)/ mouse dose of AAV-7F9-Fc results in antibody-circulating concentrations in the milligrams per milliliter range over an 8month period. Studies of locomotor activity showed that METH psychostimulant effects were decreased in potency in mice expressing AAV-7F9-Fc, and biodistribution studies showed that there were significant increases in the METH brain:serum ratio at 30 minutes after a $3.1 \mathrm{mg} / \mathrm{kg}$ METH dose.

\section{Methods and Methods}

Animal Usage. Adult (3 to 4 weeks old), male, BALB/c mice were obtained from Charles River Laboratories (Raleigh, NC). Mice were housed three to six mice per cage in a light-controlled environment (12-hour light/dark cycle). Mice were allowed food and water ad libitum. Mice were randomly placed into groups for all experimental studies. The use of mice for experiments was approved by the Institutional Care and Use Committee of the Universities of Arkansas for Medical Sciences and was in accordance with the Guide for the Care and Use of Laboratory Animals (National Research Council 2011).

Drugs and Reagents. All $S$-methamphetamine (METH) used, both unlabeled and tritium-labeled, was obtained as an $\mathrm{HCl}$ salt from the National Institute of Drug Abuse (Bethesda, MD). METH was weighed as the salt version and dissolved in $1 \times$ PBS. Unless otherwise stated, reagents and other materials were purchased from ThermoFisher Scientific (Waltham, MA). Isolation and purification of plasmids and proteins were performed using Qiagen kits (Valencia, CA). METH ampules were bought for standard curve samples and quality control samples from Cerilliant (Round Rock, TX). DNA digestion enzymes were purchased from New England Biolabs (Ipswich, MA).

Design and Generation of 7F9-Fc Plasmids and AAV8 Capsids. The scFv-Fc fusion construct, 7F9-Fc, was derived from an anti-METH mAb previously generated consisting of scFv7F9 and $\mathrm{C}_{\mathrm{H} 2}$ and $\mathrm{C}_{\mathrm{H} 3}$ regions (Peterson et al., 2008). The sequences were capped with a 6 -histidine tail (6HIS) at the carboxy terminus and expressed with a cleavable, signal secretion sequence (Human $\alpha 1$ Microglobulin/Bikunin Precursor) to ensure correct folding and routing through the cellular secretory pathway (Tyagi et al., 2002). Plasmids were cloned into cassettes with a human $\alpha-1$ antitrypsin promoter prior to the Human $\alpha 1$-Microglobulin/Bikunin Precursor sequence. The cassette structure is shown in Fig. 1A.

The sequences containing 7F9-Fc cDNA were custom synthesized and ligated into pUC57 (GenScript). Plasmids containing the antibody sequences were transformed into Invitrogen Top10F competent cells (C303003; Thermo) as per manufacturer recommendations, and sequences were confirmed by the University of Arkansas for Medical Sciences DNA Sequencing Core Facility. Plasmids were transformed into Top $10 \mathrm{~F}$ cells, and colonies of transformed cells were grown on agar plates containing $50 \mu \mathrm{g} / \mathrm{ml}$ ampicillin. Colonies were selected and added to baffled flasks containing 11 of LB with $50 \mu \mathrm{g} / \mathrm{ml}$ ampicillin. Cultures were grown overnight with shaking at $225 \mathrm{rpm}$ at $37^{\circ} \mathrm{C}$. Qiagen Endotoxin-free Mega (12381) plasmid kits were used to isolate the plasmid DNA. DNA purity and concentrations were determined by $\mathrm{UV}_{260 / 280}$ spectrophotometry and gel electrophoresis via plasmid digestions using the digestion enzymes EcoRI and XhoI (R3101S and R0146S respectively; New England Biolabs).

Plasmids containing the construct DNA were shipped to the Children's Hospital of Philadelphia to be ligated into the expression vector, AAV-human $\alpha$-1 antitrypsin-factor IX as described in Margaritis et al. (2004). These ligated expression vectors were then sent to SAB Tech (Philadelphia, PA), which employs a helper virus-free triple plasmid transfection method for AAV8 vector production and packaging into viral vectors.

Determining Titers and Duration of Expression. Four to six mice per group were administered $1 \times 10^{10}, 1 \times 10^{11}$, or $1 \times 10^{12} \mathrm{vcs}$ of AAV-7F9-Fc per mouse or equal volumes of $5 \%$ sorbitol (w/v) in $1 \times$ PBS for the vehicle group via tail vein. Tail snips were performed every 2-4 weeks to collect blood samples from each mouse (Hay et al., 2018). The serum was isolated from the red blood cells via centrifugation $\left(12,000 \mathrm{rcf}\right.$ for 10 minutes) and stored at $-80^{\circ} \mathrm{C}$.

Functional sandwich ELISAs were performed as previously described to determine the circulating concentrations of 7F9-Fc at each time point (Hay et al., 2018). Briefly, the serum samples were diluted 1:5000, and an HRP anti-His tag antibody (652504; Biolegend) diluted 1:1000 in Superblock (37515; Thermo) was used as the secondary detection antibody. Then 1-Step Ultra TMB-ELISA (34029; Thermo) was used to react with the HRP on the secondary antibodies. Wells were measured for light absorption at $480 \mathrm{~nm}$ on a Biotek HT Synergy plate reader. Concentrations of the anti-METH antibodies were interpolated from a standard curve of purified scFv7F9 protein (Reichard et al., 2016).

Biocharacterization. Western blots were performed as previously described to confirm the relative size of the expressed 7F9-Fc 
A

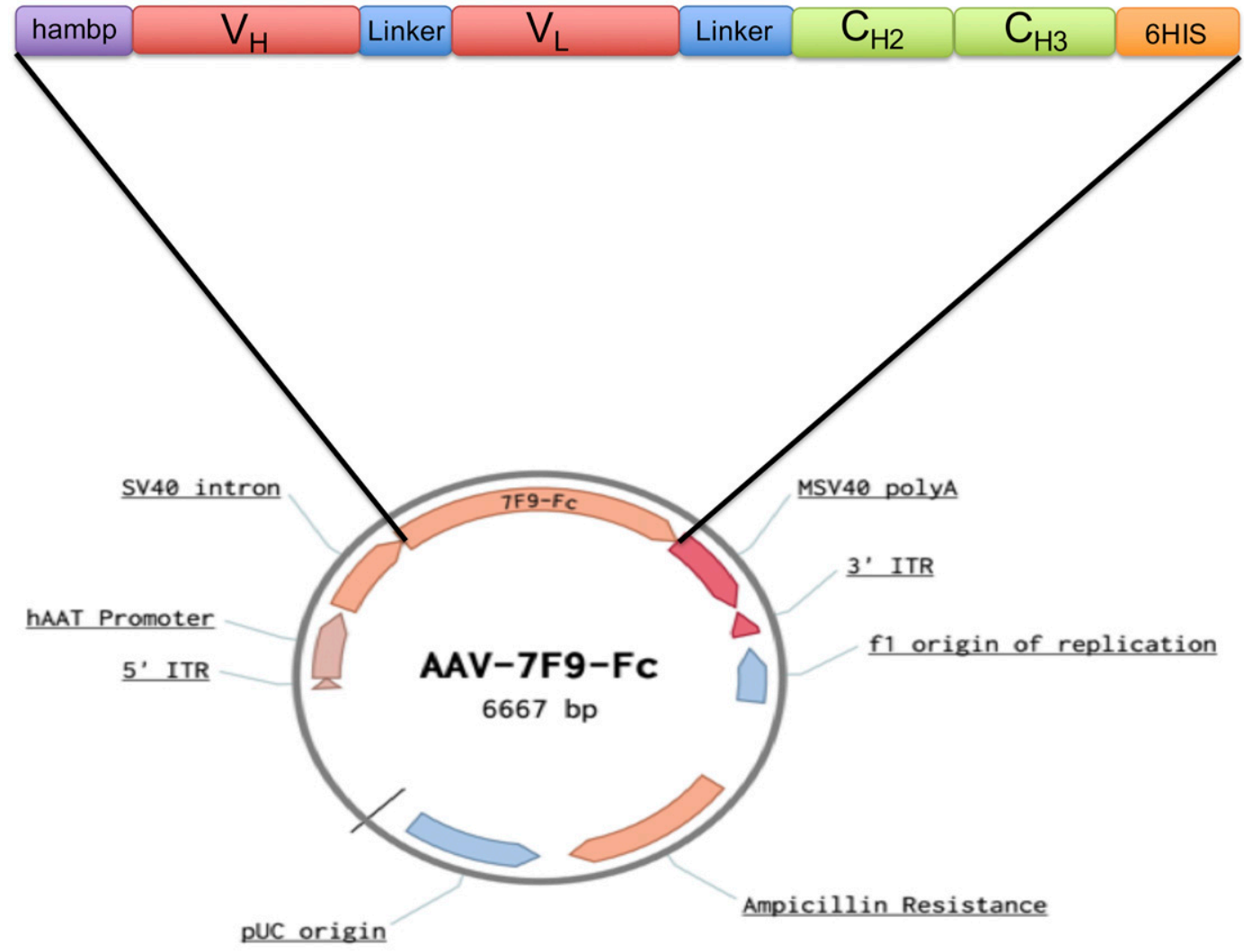

B

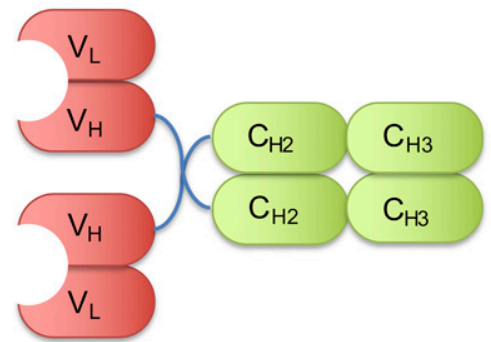

Fig. 1. Schematic of the scFv-Fc fusion construct. (A) shows a schematic of the different regions of the DNA sequence packaged into the AAV capsids. (B) shows the same sequences after being translated and folded; construct should form a homodimer before being secreted from the cell. $\mathrm{C}_{\mathrm{H} 2}$, constant region 2; $\mathrm{C}_{\mathrm{H} 3}$, constant region 3; hAAT, human $\alpha$-1 antitrypsin promoter; $6 \mathrm{His}, 6$-histidine tag for purification and identification; HMM38, secretory signal; Linker, amino acid linker; $\mathrm{V}_{\mathrm{H}}$, variable heavy region; $\mathrm{V}_{\mathrm{L}}$, variable light region.simian virus 40 (SV40), minimal SV40 promoter (mSV40), inverted terminal repeats (ITR), plasmid University of California (pUC), poly adenosine tail (polyA)

constructs (Hay et al., 2018). Briefly, serum samples collected 22 days post-AAV administration were pooled from each AAV dose group and diluted 1:13 before applying to the Western blot membranes. Serum pooled from vehicle mice on day 22 was used as a negative control to show mice not given the AAV-7F9-Fc vectors did not express 7F9-Fc protein. Purified scFv7F9 protein with a 6 His tag was used as a positive control (Reichard et al., 2016). The Western blot membranes were stained with HRP anti-His tag antibody (652504; Biolegend) diluted 1:2000 in 0.5\% (w/v) bovine serum albumin. Membranes were developed using Pierce ECL Western Blotting Substrate (32106; Thermo). Because of the use of a standard curve in the ELISA assays to precisely interpolate expression level, Western blot bands were not quantified.

Determination of AAV-7F9-Fc Antibody Ex Vivo Function. Radioimmunoassays were performed as previously described to determine the $\mathrm{IC}_{50}$ values for METH binding to 7F9-Fc (Owens et al., 1988; Peterson et al., 2007). Briefly, $10 \mu \mathrm{l}$ of serum samples or METH standards in serum (concentrations from 0.03 to $500 \mathrm{nM}$ ) were added to duplicate polypropylene tubes containing: $20 \mu \mathrm{l}$ of magnetic MagnaBind protein $\mathrm{G}$ beads (213498; Thermo), $100 \mu \mathrm{l}$ of $500 \mathrm{dpm} / \mu \mathrm{l}$ ${ }^{3} \mathrm{H}-\mathrm{METH}$, and $100 \mu \mathrm{l}$ of blank serum diluted 1:1000. After 18 hours of gentle mixing at $4^{\circ} \mathrm{C}$, the tubes were placed over magnets for 6 minutes, and the supernatant was removed. The magnetic bead/ antibody complexes were suspended in $2 \mathrm{ml}$ ScintiVerse BD Cocktail (163431; Thermo). Vials were vortexed for 10 seconds before quantitation by a Tri-Carb 2910 TR scintillation counter. An "[Inhibitor] vs. normalized response-Variable slope" model (Graphpad Prism 7.0d) was fit to the METH serum standard curve data points to determine the $\mathrm{IC}_{50}$ of each unknown serum sample.

Disposition of METH after AAV-7F9-Fc Treatment. Mice given either $1 \times 10^{12} \mathrm{vc} /$ mouse $7 \mathrm{~F} 9-\mathrm{Fc}$ therapy or vehicle were administered $3.1 \mathrm{mg} / \mathrm{kg}$ METH subcutaneously. Thirty minutes later, mice were sacrificed, and brains and trunk blood were harvested. After clotting, blood samples were centrifuged at 12,000 rcf for 10 minutes, and serum was transferred into new tubes. Brains were homogenized in $4 \mathrm{ml}$ of mass spectrometry grade $\mathrm{H}_{2} \mathrm{O}$ per gram of brain tissue. 
A previously described extraction method for (3,4)-methylenedioxypyrovalerone was adapted for the extraction of METH and AMP in serum and brain tissue (Hambuchen et al., 2017). Twenty-five microliters of each sample or standard was added to a 1.5-ml centrifuge tube. A volume of $125 \mu \mathrm{l}$ ice-cold $30 \mathrm{ng} / \mathrm{ml}$ methamphetamine-D5 (internal standard) in acetonitrile was added prior to brief vortex mixing. After adding the internal standard solution to all samples and standards, the mixtures were vortex mixed for an additional 10 seconds prior to incubation for 10 minutes at $4^{\circ} \mathrm{C}$. Afterward, the mixture was centrifuged at $20,000 \mathrm{rcf}\left(\right.$ at $\left.4^{\circ} \mathrm{C}\right)$ for 5 minutes, and the supernatant was transferred to another 1.5-ml tube for drying in a Zymark TurboVap LV evaporator (SOTAX Corporation) under gentle nitrogen flow in a water bath at $40^{\circ} \mathrm{C}$.

Dried samples and standards were reconstituted with $75 \mu \mathrm{l}$ of $0.1 \%$ formic acid and vortex mixed for 20 seconds. After reconstitution, samples were centrifuged at $20,000 \mathrm{rcf}\left(\right.$ at $\left.4^{\circ} \mathrm{C}\right)$ for 5 minutes, and $65 \mu \mathrm{l}$ of supernatant was transferred to an autoinjector plate where $7.5 \mu \mathrm{l}$ were injected onto an Acquity UPLC BEH C18 1.7- $\mu \mathrm{m}(2.1$ i.d. $\times 100 \mathrm{~mm})$ column (Waters Corp, Milford, MA) in an Acquity Ultra Performance Liquid Chromatography system connected to a Quattro Premier XE mass spectrometer (Waters Corp).

Analysis of unchanged METH concentrations in extracted samples were performed by a previously described liquid chromatography tandem mass spectrometry method (Hambuchen et al., 2015). The lower and upper limits of quantification for METH and AMP were 1 and $1000 \mathrm{ng} / \mathrm{ml}$, respectively. All predicted values for calibration and quality control standards were within $\pm 20 \%$ of the actual concentrations.

Locomotor Activity Assays in Mice. Two separate AAV-7F9-Fc locomotor studies were performed, one with $1 \times 10^{11} \mathrm{vc} /$ mouse AAV8 ( $n=8$ mice/group) and one with $1 \times 10^{12} \mathrm{vc} /$ mouse AAV8 $(n=6$ mice/ group). The $1 \times 10^{11} \mathrm{vc} /$ mouse study was performed between days 11 and 50 post-AAV administration, and the $1 \times 10^{12} \mathrm{vc} /$ mouse study was performed on the mice between days 21 and 37 post-AAV administration. Mice were given at least 21 days to achieve steady expression of 7F9-Fc in the serum before being administered drug subcutaneously. Mice were placed into $27.31 \times 27.31 \times 20.32-\mathrm{cm}$ chambers (ENV-510S; Med Associates). Chambers were equipped with IR-beam transmitter strips (ENV-256T; Med Associates) and receiver strips (ENV-256R; Med Associates) with 24 beams/strip to create a horizontal grid. Transmitter/receiver beams were in groups of three, $0.5 \mathrm{~cm}$ apart, with 1.3-cm spacing between beam triplets. Each chamber was illuminated during recording. Movement was recorded when mice interrupted beams. The software default settings for movement were used. Food and water were not available while the mice were in the chambers. Mice were allowed to equilibrate to their surroundings for 90 minutes before being administered PBS, METH or cocaine subcutaneously.

For the $1 \times 10^{11} \mathrm{vc} /$ mouse study, mice were administered saline, then $1,3,5$, and $7 \mathrm{mg} / \mathrm{kg} \mathrm{METH}$, and then 10 and $30 \mathrm{mg} / \mathrm{kg}$ cocaine subcutaneously with at least a 1 day for washout period between doses. A $10 \mathrm{mg} / \mathrm{kg}$ s.c. cocaine dose was initially used as a positive control, but this dose failed to elicit a large increase in locomotor activity over saline, so a $30 \mathrm{mg} / \mathrm{kg}$ s.c. cocaine dose was also administrered. For the $1 \times 10^{12} \mathrm{vc} /$ mouse study, mice were given $1.7,3.1$, and $9.4 \mathrm{mg} / \mathrm{kg}$ METH subcutaneously with at least a 1-day washout period in between doses. These doses were prepared as 1.0, 3.0 , and $10 \mathrm{mg} / \mathrm{kg} \mathrm{METH}$, respectively, but afterward were validated by LC-MS/MS and found to be slightly different from the target doses. For testing days, drug stocks were prepared such that an injection volume of $100 \mu \mathrm{l}$ would deliver the desired dose to a mouse based on the mean weight of the group. The weight variation between individuals was less than $6 \%$. The corrected values were used for all data analysis.

After drug administration, mice were placed back into their beam break boxes. Recording started once the mouse was placed in the box. Mouse activity was recorded for 2 hours postinjection. Afterward, mice were placed back into their home cages. There was at least a 2-day washout period between testing sessions. Activity was recorded as distance in $\mathrm{cm}$ and was reported as the total distance traveled over 90 minutes postadministration of METH.

Statistical Analyses. GraphPad Prism (version 7.0d; GraphPad Software, Inc.) was used to analyze all data. To determine appropriate $n$ values before each study, power analyses were performed using the http://clincalc.com/Stats/SampleSize.aspx website with power set to 0.8 and $\alpha=0.05$. No outliers were identified in the data (Grubb's outlier test, $\alpha=0.05$ ). Shapiro-Wilk normalcy tests were used to confirm data were normally distributed. The resulting data from the METH biodistribution study were analyzed via one-tailed, unpaired $t$ tests. Unless otherwise noted, significance was determined at $P<$ 0.05 and denoted with an asterisk.

For the $1 \times 10^{12} \mathrm{vc} /$ mouse dose locomotor results, the comparisons of the peak circulating concentrations of the construct between the different doses of AAV were tested with a one-way ANOVA using a Holm-Sidak post hoc multiple comparison test when a significant difference was found between groups. After statistical significance was observed in the interaction between METH dose and AAV therapy for the locomotor assays with a two-way ANOVA, simple main effect tests with a HolmSidak correction were performed to further assess interaction. HolmSidak multiple comparisons tests were also performed to compare effects of METH doses to saline doses. Data are reported as percent saline.

The locomotor activity results for the $1 \times 10^{11} \mathrm{vc} / \mathrm{mouse}$ dose used a different set of analyses. The results were normalized by setting the smallest mean in each data set as $0 \%$ and the largest data set as $100 \%$. Then a nonlinear regression model using an "[Agonist] vs. normalized response" equation was used to determine the $\mathrm{EC}_{50}$ 's of METH for mice given the AAV-7F9-Fc therapy and mice given vehicle. To show there was no difference in the efficacy of METH between treatment groups, the AUCs of each individual mouse were determined, and an unpaired $t$ test was performed. Data are reported as percent saline.

\section{Results}

Determining Optimal AAV Dose for 7F9-Fc Serum Expression. A dose-dependent relationship was observed in Fig. 2A between the dose of AAV-7F9-Fc administered and the circulating concentration of anti-METH antibodies. Over the observed 170-day period, the mice administered $1 \times 10^{10} \mathrm{vc} /$ mouse peaked at $48 \mu \mathrm{g} / \mathrm{ml}$, those administered $1 \times 10^{11} \mathrm{vc} /$ mouse peaked at $1785 \mu \mathrm{g} / \mathrm{ml}$, and those administered $1 \times 10^{12}$ $\mathrm{vc} /$ mouse peaked at $3831 \mu \mathrm{g} / \mathrm{ml}$. A one-way ANOVA of the peak values yielded significant variation between groups $\mathrm{F}(2,12)=2.058, P<0.0001$. Tukey post hoc tests showed there were significant differences in expression between each of the observed peak circulating concentrations $(P<0.05)$.

Confirmation of Construct Size. Serum samples collected from mice on day 22 post-AAV administration were pooled by AAV dose (vehicle, $1 \times 10^{10}, 1 \times 10^{11}$, or $1 \times 10^{12} \mathrm{vc} /$ mouse). The 7F9-Fc protein monomers were calculated to be $61.6 \mathrm{kDa}$. The intact monomeric 7F9-Fc bands appeared at the expected molecular size (60 kDa, Fig. 2B). As observed in Fig. 2B, the 7F9-Fc bands appear to increase in intensity with increasing $\mathrm{AAV}$ dose, but the band for the lowest concentration is not detectable. This is likely due to the very low 7F9-Fc concentration of the $1 \times 10^{10}$ dose reported by ELISA in Fig. 2A.

Affinity. Pooled serum samples from mice expressing 7F9Fc collected 22 days after AAV administration showed the $\mathrm{IC}_{50}$ value for $7 \mathrm{~F} 9-\mathrm{Fc}$ was $17 \mathrm{nM}$, whereas the $\mathrm{mAb7F} \mathrm{IC}_{50}$ value was $32 \mathrm{nM}$ (Fig. 3).

Duration of Anti-METH Antibody Expression in Mice. We selected the highest $1 \times 10^{12} \mathrm{vc} /$ mouse dose of AAV-7F9-Fc for further studies. An additional mouse cohort 
A

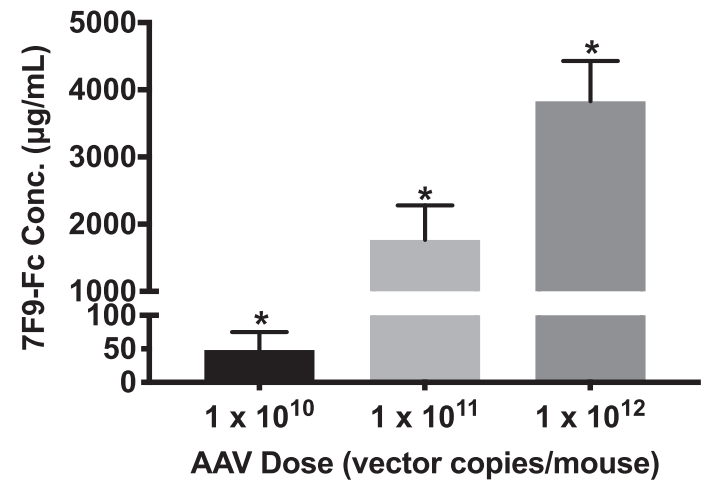

B

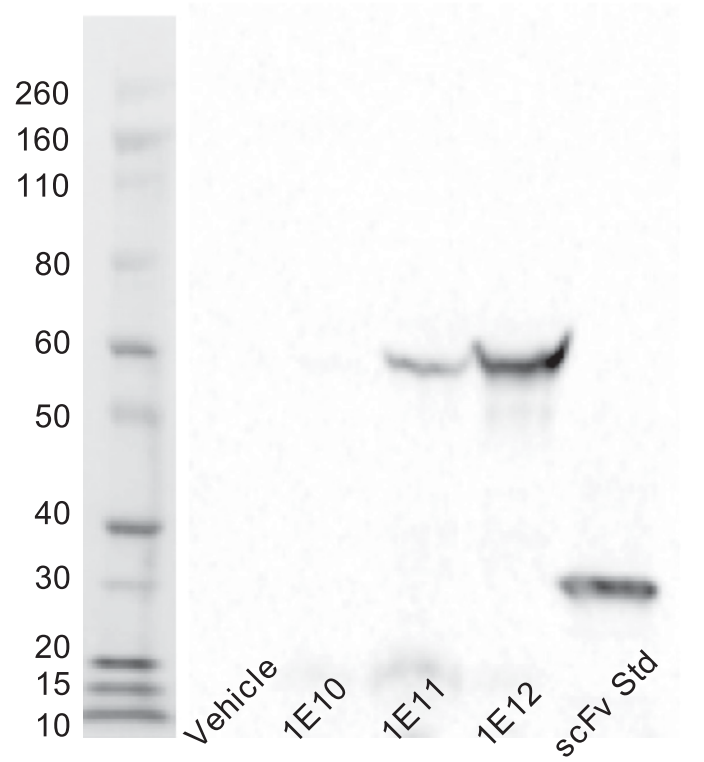

Fig. 2. A dose-dependent relationship exists between the dose of AAV given to mice and the level of expression of 7F9-Fc. (A) Peak serum concentration of 7F9-Fc as determined by functional ELISA $(* P<0.05)$. Data shown as mean \pm S.D., $n=8$ per group. (B) A Western blot comparing the expressed size of construct $(61 \mathrm{kDa})$ to that of a predecessor scFv standard of known size $(23 \mathrm{kDa})$.

administered this dose to determine the longevity of 7F9-Fc expression in vivo over an $\sim 8$-month observation period. As can be observed in Fig. 4, the mice expressed 7F9-Fc antiMETH antibodies for at least 239 days after a single AAV administration. During the observation period, the mice exhibited an average circulating concentration of $2116 \pm$ $1543 \mu \mathrm{g} / \mathrm{ml}$ with a peak of $5228 \pm 1637 \mu \mathrm{g} / \mathrm{ml}$ on day 63 post-AAV administration.

Locomotor Studies. At the $1 \times 10^{12} \mathrm{vc} / \mathrm{mouse}$ dose, the AAV-7F9-Fc therapy decreased the psychostimulant effects of $\mathrm{METH}$ at 3.1 and $9.4 \mathrm{mg} / \mathrm{kg}$ s.c. METH doses (Fig. 5A) as compared with vehicle mice. A two-way ANOVA on the total distance traveled over a 90-minute period revealed a statistically significant main effect of whether the therapy was administered $[\mathrm{F}(1,8)=20.03]$ a statistically significant main effect of the METH dose $[\mathrm{F}(4,32)=9.384]$, and the interaction between METH dose and AAV therapy was also significant $[\mathrm{F}(4,32)=5.114]$. A Holm-Sidak post hoc test revealed significant difference between vehicle mice and mice treated

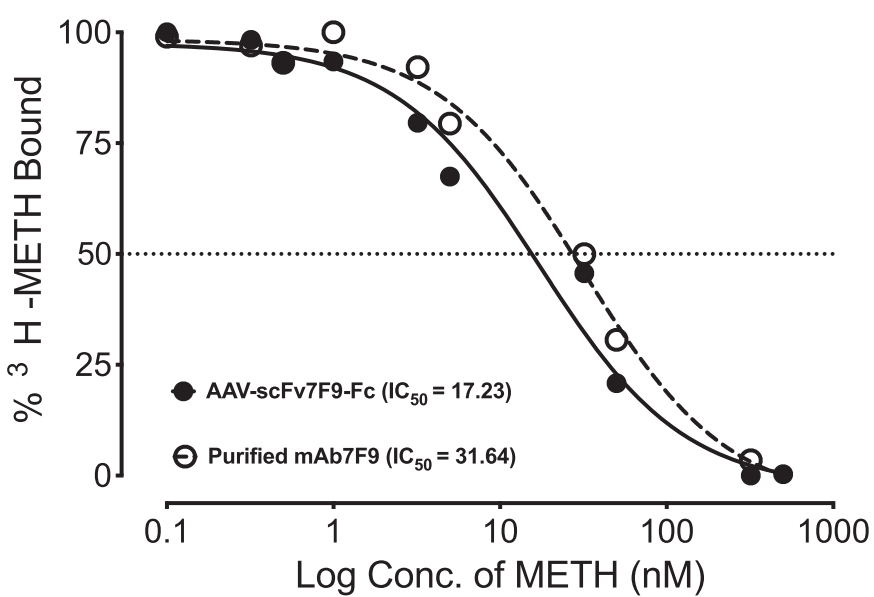

Fig. 3. Comparison of $\mathrm{IC}_{50}$ values for METH between culture-produced mAb7F9 and mouse-expressed 7F9-Fc. The $\mathrm{IC}_{50}$ (nanomolar) for all variants was estimated at $50 \%{ }^{3} \mathrm{H}$-METH bound (dotted line). Data shown as mean \pm S.D., $n=8$ per group.

with the AAV-7F9-Fc therapy at the 3.1 and $9.4 \mathrm{mg} / \mathrm{kg}$ s.c. METH doses and also showed that there was no statistically significant difference in total distrance traveled between the METH doses and the saline doses in the mice treated with the AAV-7F9-Fc therapy. In all experimental groups, administration of saline had no effects on mouse behavior, and locomotor activity remained low for all subjects.

There was nearly a 2 -fold shift to the right in the $\mathrm{ED}_{50}$ of METH for the mice administered $1 \times 10^{11} \mathrm{vc} / \mathrm{mouse}$ of the AAV-7F9-Fc therapy $\left(\mathrm{ED}_{50}=3.2 \mathrm{mg} / \mathrm{kg}\right)$ compared with the vehicle mice $\left(\mathrm{ED}_{50}=1.7\right) \mathrm{mg} / \mathrm{kg}$, as can be seen in Fig. 5B. The data observed in Fig. 5B show that the AAV-7F9Fc therapy can still reduce the potency of METH by nearly 2-fold at $48 \%$ of the average circulating $7 \mathrm{~F} 9-\mathrm{Fc}$ concentrations resulting from the $1 \times 10^{12} \mathrm{vc} / \mathrm{mouse}$ dose. There was not a significant difference in the observed maximal effectiveness of METH between the two groups $(P=0.0678)$. These mice exhibited an

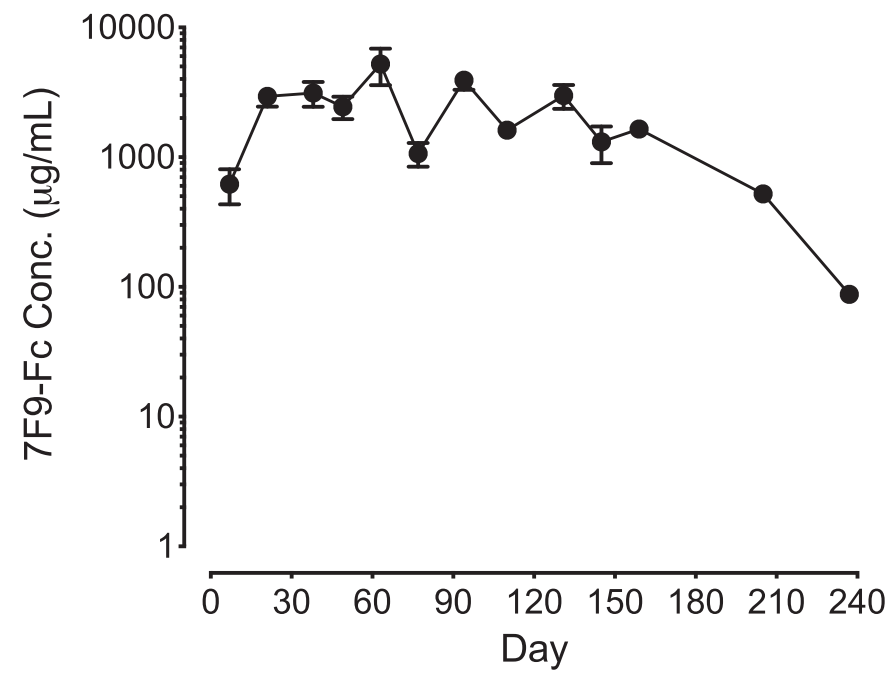

Fig. 4. 7F9-Fc expression persists over 8 months. Mice given $1 \times 10^{12}$ vector copies/mouse showed expression for at least 8 months, with an average expression of $2100 \mu \mathrm{g} / \mathrm{ml}$. Functional ELISAs were performed on mouse serum. Data given as mean \pm S.D., $n=6$ per group. 

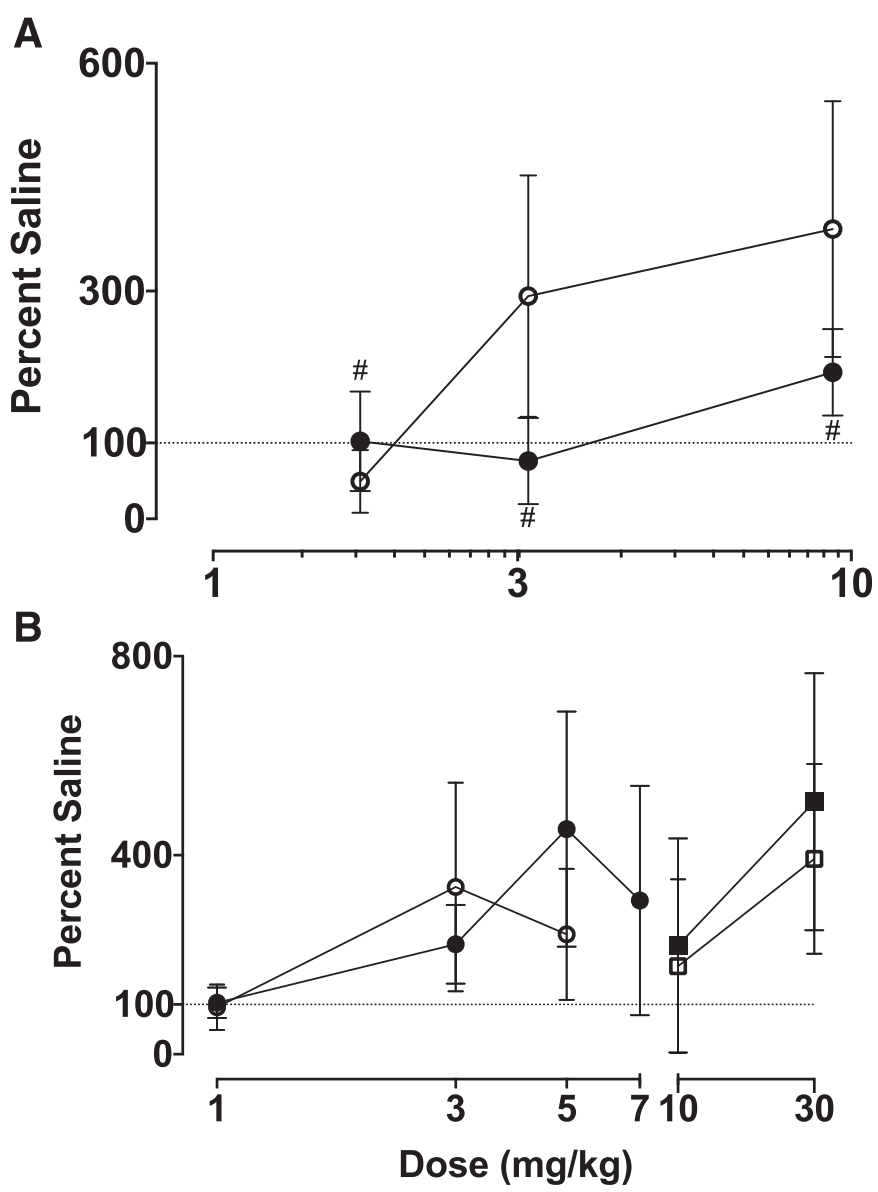

๑ Vehicle METH घ Vehicle Cocaine - 7F9-Fc METH = 7F9-Fc Cocaine

Fig. 5. 7F9-Fc significantly reduces the psychostimulant effects of METH. Two groups of mice, vehicle and mice given (A) $1 \times 10^{12} \mathrm{vc} \mathrm{AAV-}$ $7 \mathrm{~F} 9-\mathrm{Fc} /$ mouse and (B) $1 \times 10^{11}$ vc AAV-7F9-Fc/mouse, were either administed saline or increasing doses of METH subcutaneously. The locomotor activity measured as total distance traveled was recorded for 90 minutes. Comparison between AAV-7F9-Fc-treated mice $(\# P<0.05)$. Comparison between vehicle and AAV-7F9-Fc mice $(* P<0.05)$. Data are shown as mean \pm S.D., $n=6(\mathrm{~A})$ and $n=8$ (B) per group.

average 7F9-Fc circulating concentration of $230 \mu \mathrm{g} / \mathrm{ml}$ before testing began (62\% lower than the $1 \times 10^{12}$ group).

Physiologic Efficacy. Four months postadministration of either AAV or vehicle, the same mice used in the locomotor assays were given $3.1 \mathrm{mg} / \mathrm{kg}$ METH s.c. As can be observed in Fig. 6A, mice treated with AAV-7F9-Fc accumulated significantly less $(P=0.03) \mathrm{METH}$ in their brains $(2407 \mathrm{ng} \mathrm{METH} / \mathrm{g}$ brain) than vehicle mice (2863 ng METH/g brain). This equates to a 1.2-fold decrease in METH concentration in the brains of the treated mice. In the serum (Fig. 6B), the treated mice sequestered significantly more $(P=0.0053) \mathrm{METH}$ in their serum (828 $\mathrm{ng} \mathrm{METH} / \mathrm{ml}$ serum) than vehicle mice (324 ng METH/ml serum), which equates to a 2.5-fold increase in METH in the serum of the treated mice.

Mouse Health. Similarly to our previously reported data with the same concentration of AAV, no lasting adverse effects of either AAV administration or of 7F9-Fc expression were observed as a result of the high AAV dose (Hay et al., 2018).

\section{Discussion}

The purpose of this study was to design and test both in vivo and ex vivo a long-lasting AAV-delivered anti-METH antibody therapy. The overarching hypothesis was that continuous expression of a high-affinity anti-METH antibody with a longlasting serum residence time could be a viable approach for creating medications to aid in treatment of METH-use disorders. We have previously presented proof-of-concept data on expression of such an approach with an anti-METH mAb transgene (Hay et al., 2018). Here, we wanted to increase the transgene expression and provide data on its functional capacity against METH effects. For this purpose, we engineered a single gene sequence based on a high-affinity monoclonal antibody (mAb7F9) that could efficiently be packaged into AAV8 particles. By incorporating an Fc region into the construct, detectable expression lasted at least another month with average expression levels of $2100 \mu \mathrm{g} / \mathrm{ml}$. This produced 30 times greater concentrations than the first-generation constructs, which was likely due to significantly reduced clearance of scFv-7F9-Fc (Hay et al., 2018).

Three doses of the AAV-7F9-Fc therapy $\left(1 \times 10^{10}, 1 \times 10^{11}\right.$, and $1 \times 10^{12} \mathrm{vc} / \mathrm{mouse}$ ) were used to determine dose-response effects on serum concentrations of expressed scFv-7F9-Fc. Because a significantly higher circulating concentration of our anti-METH construct at the $1 \times 10^{12} \mathrm{vc} /$ mouse dose was detected, we chose this dose to determine the potential upper limit of reduction of the pharmacological effects of METH. Future studies will be required to determine what dose of the AAV-7F9-Fc therapy provides the optimal circulating concentrations for the AAV dose given.

As can be observed in Fig. 5A, locomotor activity after administration of the $9.4-\mathrm{mg} / \mathrm{kg}$ METH dose for the treated mice, although not significantly greater than activity observed after the saline doses, appears to be visually greater than the activity quantified after treatment with either of the two lower METH doses. Although a more extensive panel of behavioral assays would be required to confirm, the data appear to suggest that the AAV-7F9-Fc-treated mice are beginning to exhibit psychostimulant effects of METH around the $9.4 \mathrm{mg} / \mathrm{kg}$ METH dose compared with the vehicle mice beginning to exhibit the psychostimulant effects of METH between the 1.7 and $3.1 \mathrm{mg} / \mathrm{kg}$ METH doses. Using linear regressions, the treated mice required about 3.8 times more METH to exhibit the same level of activity at the $9.4 \mathrm{mg} / \mathrm{kg}$ s.c. METH dose. This indicates that the $1 \times 10^{12} \mathrm{vc} /$ mouse dose of AAV-7F9-Fc decreases the potency of METH nearly 4-fold.

To better understand the dose-effect relationship between 7F9-Fc circulating concentrations and psychostimulant effects of METH, a second locomotor assay was performed with mice administered $1 \times 10^{11}$ AAV-7F9-Fc vc/mouse. We observed that both treated and vehicle mice exhibited a classic inverted "U" dose-reponse curve. We were unable to observe a descending limb in dose-effect curves in the $1 \times 10^{12} \mathrm{vc} / \mathrm{mouse}$ locomotor study because about half of the mice began exhibiting adverse METH effects at $16 \mathrm{mg} / \mathrm{kg}$, and the assay was terminated. Because descending limbs could be recorded for the $1 \times 10^{11}$ AAV-7F9-Fc vc/mouse dose locomotor study, a different, more descriptive set of calculations could be performed to explain these results. This locomotor study also suggests that, although the AAV-7F9-Fc therapy can reduce the potency of METH, it might not be completely effective at 
A

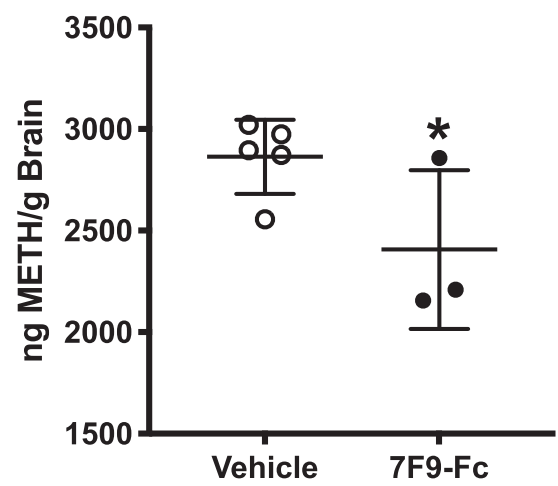

B

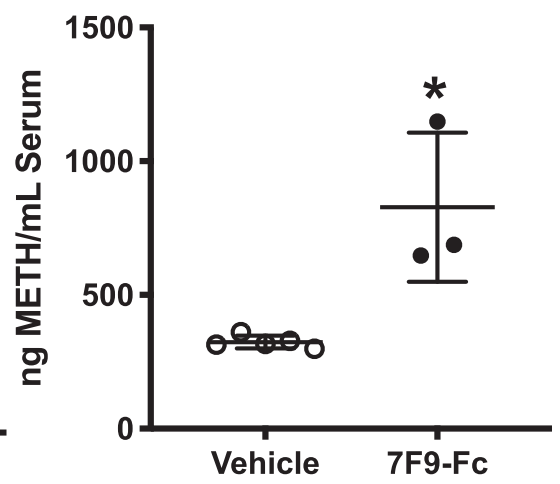

Fig. 6. A comparison of METH brain and serum concentrations after a $3.1 \mathrm{mg} / \mathrm{kg}$ s.c. injection of METH. Two groups of mice, vehicle and mice given $1 \times 10^{12} \mathrm{vc}$ AAV-7F9-Fc/mouse, were administered $3.1 \mathrm{mg} / \mathrm{kg}$ METH s.c. Thirty minutes after METH administration, mice were sacrificed, and brains (A) and serum (B) were collected. METH concentrations were determined by liquid chromatography tandem mass spectrometry $(* P<0.05)$. Data points are shown as mean \pm S.D., $n=3-5$ per group. reducing all of METH's adverse effects at extremely high doses (i.e., once the antibodies have exceeded full occupancy). This is similar to other antagonists that can be surmounted with extremely high drug doses, such as naltrexone for opioid-use disorders.

We acknowledge that the METH locomotor activity doseresponse curves in Fig. 5 do not neatly match up above the $3 \mathrm{mg} / \mathrm{kg}$ s.c. METH doses. The mice administered $1 \times 10^{11} \mathrm{vc} /$ mouse of AAV-7F9-Fc were more active overall than the mice in the $1 \times 10^{12} \mathrm{vc} / \mathrm{mouse}$ locomotor study. The two locomotor studies were performed over 2 years apart, but other variables were matched as closely as possible including the mouse vendor, the age of the mice when performing the studies, and the experimental setup. In an attempt to reduce baseline activity, the box habituation time was increased, and additional saline sessions were run, but the $1 \times 10^{11} \mathrm{vc} /$ mouse mice continued to exhibit more activity than the $1 \times 10^{12} \mathrm{vc} / \mathrm{mouse}$ mice. Nevertheless, the decreased locomotor effects of methamphetamine observed at doses above $3.0 \mathrm{mg} / \mathrm{kg}$ for control mice in Fig. 5B (consistent with induction of motor stereotypy) were not observed for control mice in Fig. 5A, even at a 3-fold higher dose. We can only attribute this to nonspecific cohort effects. Despite this acknowledged cohort difference, a very consistent locomotor effect of 3.0 or $3.1 \mathrm{mg} / \mathrm{kg} \mathrm{METH}$ was observed in both studies, in which activity was increased to approximately $300 \%$ of saline control in vehicle mice. Importantly, the AAV-7F9-Fc therapy dose-dependently decreased these METH effects to approximately $200 \%$ of saline control in the $1 \times 10^{11} \mathrm{vc} / \mathrm{mouse}$ group and to approximately $100 \%$ of saline control in the $1 \times 10^{12} \mathrm{vc} /$ mouse group.

As previously noted, other groups (Hicks et al., 2012; Rosenberg et al., 2012, 2013) have successfully expressed full IgG antibodies using gene transfer. Although this approach is viable, we hypothesized that a simpler approach might result in higher circulating concentrations due to less cellular processing. The scFv-Fc fusion construct can be expressed as a single continuous chain, which does not require posttranslational cleavage steps for assembly (Unverdorben et al., 2016). Furthermore, scFv-Fc fusion constructs were previously shown to have similar half-lives compared with full IgG antibodies, primarily because of similar sizes and use of the neonatal Fc receptor pathway, which greatly increases the half-life of proteins (e.g., antibodies) beyond what would be predicted for proteins of their size (Ghetie et al., 1996).

We also chose to use the $\operatorname{IgG}_{2}$ subclass rather than the $\operatorname{IgG}_{1}$ subclass because others have (Hicks et al., 2012; Rosenberg et al., 2012). The $\operatorname{IgG}_{2}$ subclass has a similar affinity to the neonatal $\mathrm{Fc}$ receptor, but $\mathrm{IgG}_{2}$ has a lower affinity for the Fc receptors on effector cells and is far less effective at activating complement (Vidarsson et al., 2014), which was not needed in our treatment modality. These differences could translate to a decrease in undesirable systemic immune responses as a result of the expressed anti-METH antibodies.

Although the variable region for both of the 7F9-Fc and mAb7F9 standard proteins is the same, the Fc regions of the protein are different, which perhaps could explain the difference in affinity ( $17 \mathrm{nM}$ vs. $32 \mathrm{nM}$ ). This pattern was observed within the scFv constructs of scFv7F9 showing an affinity to METH of $22 \mathrm{nM}$ compared with an affinity of $48 \mathrm{nM}$ of the purified standard (Hay et al., 2018). This is the first time our group has compared the affinity of the 7F9-Fc construct to the original IgG mAb7F9 within the same assay. The overlapping locomotor effects in treated and untreated mice in response to the 10 and $30 \mathrm{mg} / \mathrm{kg}$ s.c. cocaine administration in the $1 \times 10^{11}$ vc/mouse locomotor study (Fig. 5B) suggest that the AAV-7F9Fc therapy is selective to METH. Furthermore, the similarity in responses between treated and untreated mice to both cocaine doses may suggest that the AAV-7F9-Fc therapy does not affect central dopaminergic pathways that mediate locomotor effects of psychostimulants or nonspecifically alter drug metabolism. The AAV-7F9-Fc dose-dependent decreases in METH-elicited locomotor stimulation are therefore consistent with decreased METH concentrations in the central nervous system and increased METH sequestration in the serum, as demonstrated in Fig. 6.

The data shown in Fig. 6 indicate that 30 minutes post-METH administration, approximately $\mathrm{T}_{\max }$ for $\mathrm{METH}$ in mice brain and serum (Magyar et al., 2007), significantly greater concentrations of METH were being sequestered in the serum of the treated mice. When this study was performed (day 129) the antibodycirculating concentration of $87 \mu \mathrm{g} / \mathrm{ml}$ resulted in a METH concentration of $827 \pm 279 \mathrm{ng} / \mathrm{ml}$ in the serum. This resulted in a METH:7F9-Fc molar ratio of 1561:1 (5.54 mM METH and 3.55 $\mu \mathrm{M}$ 7F9-Fc), but even with this low antibody concentration, we observed sequestration of METH in the serum and reduction in brains of the treated mice compared with the vehicle mice. Had the assay been performed shortly after the mice had finished the locomotor assay with concentrations of about $600 \mu \mathrm{g} / \mathrm{ml}$ (unpublished data), the reduction of METH in the brains would likely have been much greater. This dose of METH was selected to challenge the circulating antibodies, and it showed that even after the antibody is surmounted, physiologic efficacy can be observed. 
In conclusion, our studies show the design, expression, and preclinical characterization of a high-affinity, long duration of action anti-METH antibody-based gene therapy. The incorporation of the Fc region of the antibody fragment resulted in sustained circulating serum concentrations of over $2.1 \mathrm{mg} / \mathrm{kg}$ in mouse serum over a period of almost 8 months. Biodistribution characterization suggests that the therapy was capable of sequestering METH in the serum and out of the brain. Data from behavioral assays suggest that this anti-METH gene therapy decreases the potency of METH to elicit locomotor stimulant effects by 2 - to 4 -fold, depending on the dose of AAV administered. Taken together, these initial studies suggest that a scFv-Fc-based AAV therapy can decrease the potency the stimulant effects of METH by reducing the amount of METH able to enter the brain in mice.

\section{Acknowledgments}

We would like to thank Melinda Gunnell, Michael Berquist II, Madison Berg, and Robin Mulkey for their expert technical assistance.

\section{Authorship Contributions}

Participated in research design: Hay, Peterson.

Conducted experiments: Hay, Ewing, Hambuchen, Zintner, Small, Bolden.

Contributed new reagents or analytic tools: Fantegrossi, Margaritis, Owens, Peterson.

Performed data analysis: Hay, Hambuchen, Peterson.

Wrote or contributed to the writing of the manuscript: Hay, Ewing, Hambuchen, Zintner, Small, Fantegrossi, Margaritis, Owens, Peterson.

\section{References}

Artigiani EE, Hsu MH, McCandlish D, and Wish ED (2019) Methamphetamine a regional drug crisis. National Drug Early Warning System, College Park, MD.

Brecht M-L and Herbeck D (2014) Time to relapse following treatment for meth amphetamine use: a long-term perspective on patterns and predictors. Drug Alcohol Depend 139:18-25.

Fang J, Qian J-J, Yi S, Harding TC, Tu GH, VanRoey M, and Jooss K (2005) Stable antibody expression at therapeutic levels using the 2A peptide. Nat Biotechnol 23 : $584-590$.

Ghetie V, Hubbard JG, Kim JK, Tsen MF, Lee Y, and Ward ES (1996) Abnormally short serum half-lives of IgG in beta 2-microglobulin-deficient mice. Eur $J$ Immunol 26:690-696.

Hambuchen MD, Carroll FI, Rüedi-Bettschen D, Hendrickson HP, Hennings LJ, Blough BE, Brieaddy LE, Pidaparthi RR, and Owens SM (2015) Combining active immunization with monoclonal antibody therapy to facilitate early initiation of a long-acting anti-methamphetamine antibody response. $J$ Med Chem 58: 4665-4677.

Hambuchen MD, Hendrickson HP, and Owens SM (2017) Chiral determination of 3,4-methylenedioxypyrovalerone enantiomers in rat serum. Anal Methods 9: 609-617.

Hay CE, Gonzalez GA III, Ewing LE, Reichard EE, Hambuchen MD, NanawareKharade N, Alam S, Bolden CT, Owens SM, Margaritis P, et al. (2018)
Development and testing of AAV-delivered single-chain variable fragments for the treatment of methamphetamine abuse. PLoS One 13:e0200060.

Hicks MJ, Rosenberg JB, De BP, Pagovich OE, Young CN, Qiu J, Kaminsky SM, Hackett NR, Worgall S, Janda KD, Davisson RL, and Crystal RG (2012) AAVdirected persistent expression of a gene encoding anti-nicotine antibody for smoking cessation. Sci Transl Med 4:140ra87.

Ho SCL, Bardor M, Li B, Lee JJ, Song Z, Tong YW, Goh L-T, and Yang Y (2013) Comparison of internal ribosome entry site (IRES) and Furin-2A (F2A) for monoclonal antibody expression level and quality in CHO cells. PLoS One 8:e63247.

Magyar K, Szatmáry I, Szebeni G, and Lengyel J (2007) Pharmacokinetic studies of (-)-deprenyl and some of its metabolites in mouse. J Neural Transm Suppl $165-173$.

Margaritis P, Arruda VR, Aljamali M, Camire RM, Schlachterman A, and High KA (2004) Novel therapeutic approach for hemophilia using gene delivery of an engineered secreted activated Factor VII. J Clin Invest 113:1025-1031.

McMillan DE, Hardwick WC, Li M, and Owens SM (2002) Pharmacokinetic antagonism of (+)-methamphetamine discrimination by a low-affinity monoclonal antimethamphetamine antibody. Behav Pharmacol 13:465-473.

Nanaware-Kharade N, Thakkar S, Gonzalez GA III, and Peterson EC (2015) A nanotechnology-based platform for extending the pharmacokinetic and binding properties of anti-methamphetamine antibody fragments. Sci Rep 5:12060.

Owens SM, Zorbas M, Lattin DL, Gunnell M, and Polk M (1988) Antibodies against arylcyclohexylamines and their similarities in binding specificity with the phencyclidine receptor. J Pharmacol Exp Ther 246:472-478.

Peterson EC, Gunnell M, Che Y, Goforth RL, Carroll FI, Henry R, Liu H, and Owens SM (2007) Using hapten design to discover therapeutic monoclonal antibodies for treating methamphetamine abuse. J Pharmacol Exp Ther 322:30-39.

Peterson EC, Laurenzana EM, Atchley WT, Hendrickson HP, and Owens SM (2008) Development and preclinical testing of a high-affinity single-chain antibody against (+)-methamphetamine. J Pharmacol Exp Ther 325:124-133.

Reichard EE, Nanaware-Kharade N, Gonzalez GA III, Thakkar S, Owens SM, and Peterson EC (2016) PEGylation of a high-affinity Anti-(+)Methamphetamine single chain antibody fragment extends functional half-life by reducing clearance. Pharm Res 33:2954-2966.

Repp R, Kellner C, Muskulus A, Staudinger M, Nodehi SM, Glorius P, Akramiene D, Dechant M, Fey GH, van Berkel PHC, et al. (2011) Combined Fc-protein- and Fcglyco-engineering of $\mathrm{scFv}-\mathrm{Fc}$ fusion proteins synergistically enhances CD16a binding but does not further enhance NK-cell mediated ADCC. J Immunol Methods 373:67-78.

Rosenberg JB, De BP, Hicks MJ, Janda KD, Kaminsky SM, Worgall S, and Crystal RG (2013) Suppression of nicotine-induced pathophysiology by an adenovirus hexon-based antinicotine vaccine. Hum Gene Ther 24:595-603.

Rosenberg JB, Hicks MJ, De BP, Pagovich O, Frenk E, Janda KD, Wee S, Koob GF, Hackett NR, Kaminsky SM, et al. (2012) AAVrh.10-mediated expression of an anticocaine antibody mediates persistent passive immunization that suppresses cocaine-induced behavior. Hum Gene Ther 23:451-459.

Tyagi S, Salier J-P, and Lal SK (2002) The liver-specific human alpha(1)-microglobulin/bikunin precursor (AMBP) is capable of self-association. Arch Biochem Biophys 399:66-72.

Unverdorben F, Richter F, Hutt M, Seifert O, Malinge P, Fischer N, and Kontermann $\mathrm{RE}$ (2016) Pharmacokinetic properties of $\mathrm{IgG}$ and various Fc fusion proteins in mice. MAbs 8:120-128.

Vidarsson G, Dekkers G, and Rispens T (2014) IgG subclasses and allotypes: from structure to effector functions. Front Immunol 5:520.

Winkelman TNA, Admon LK, Jennings L, Shippee ND, Richardson CR, and Bart G (2018) Evaluation of amphetamine-related hospitalizations and associated clinical outcomes and costs in the United States. JAMA Netw Open 1:e183758.

Zolotukhin S (2005) Production of recombinant adeno-associated virus vectors. Hum Gene Ther 16:551-557.

Address correspondence to: Dr. Eric C. Peterson, University of Arkansas for Medical Sciences, Department of Pharmacology and Toxicology 4301 West Markham St., Slot 611, Little Rock, AR 72205. E-mail: epeterson@uams.edu 